\title{
Efecto del tratamiento y tiempo de almacenamiento sobre los compuestos funcionales de subproductos de mora y fresa
}

\section{Effect of treatment and storage time on the functional compounds of blackberry and strawberry by-products}

\author{
Leidy Aguirre ${ }^{1}$; Laura Cubillos ${ }^{2}$; Martha Tarazona-Díaz ${ }^{3}$; Ligia Rodriguez ${ }^{4}$
}

11.ng. Alimentos. Universidad de Bogotá Jorge Tadeo Lozano, e-mail: leidyf.aguirrer@utadeo.edu.co, Dhttps://orcid.org/0000-0002-2992-3349

${ }^{2}$ Ing. Alimentos. Universidad de Bogotá Jorge Tadeo Lozano, e-mail: lauran.cubillosp@utadeo.edu.co, Dhttps://orcid.org/0000-0003-4318-3727

${ }^{3}$ Ing. Alimentos, Ph.D., Programa de Ingeniería de Alimentos. Universidad de Bogotá Jorge Tadeo Lozano, e-mail: marthap.tarazonad@utadeo.edu.co, (1Dhttps://orcid.org/0000-0003-0871-7295

${ }^{4}$ Ing. Alimentos, M.Sc., Programa de Ingeniería de Alimentos. Universidad de Bogotá Jorge Tadeo Lozano, e-mail: ligia.rodriguez@utadeo.edu.co, Dhttps:// orcid.org/0000-0003-2258-2155

Cómo citar: Aguirre, L.; Cubillos, L.; Tarazona-Díaz, M.; Rodriguez, L. 2019. Efecto del tratamiento y tiempo de almacenamiento sobre los compuestos funcionales de subproductos de mora y fresa. Rev. U.D.C.A Act. \& Div. Cient. 22(1):e1169. https://doi.org/10.31910/ rudca.v22.n1.2019.1169

Artículo de acceso abierto publicado por Revista U.D.C.A Actualidad \& Divulgación Científica, bajo una licencia Creative Commons CC BY-NC 4.

Recibido: Junio 18 de 2018

Aceptado: Febrero 19 de 2019

\section{RESUMEN}

Cerca de un tercio de las partes comestibles de los alimentos para el consumo humano se pierden, siendo mermas asociadas a factores de toda la cadena de abastecimiento. Dentro de dichos alimentos, se destacan la fresa (Fragaria ananassa) y la mora (Rubus glaucus Benth), frutas que han demostrado alto poder antioxidante, siendo relacionado con la prevención o el tratamiento de enfermedades. Por consiguiente, con el propósito de evidenciar el potencial para la obtención de compuestos de interés en los subproductos del procesamiento de dichas frutas, el presente estudio, se enfocó en la caracterización de los parámetros fisicoquímicos, color, poder antioxidante y vitamina $\mathrm{C}$, en cuatro condiciones de conservación, durante cuatro semanas; estas fueron: coproducto fresco, refri- gerado, secado convencional y liofilización, siendo caracterizadas también por su contenido de minerales. A partir de los resultados, se evidenció baja concentración de sólidos solubles, $\mathrm{pH}$ variable, entre 3,2 y 6,4, así como parámetros de color estables, durante el almacenamiento. Adicionalmente, los coproductos con mayor poder antioxidante fueron la semilla de mora liofilizada y el lodo de mora por secado convencional, superando al residuo de fresa en cualquier condición, según los ensayos FRAP y DPPH, respectivamente; no obstante, todas las muestras presentaron bajos contenidos en vitamina $C$, mientras que se caracterizaron por su alto contenido en potasio y hierro. Por consiguiente, lo anterior, muestra el interés por la obtención de compuestos, como minerales y antioxidantes, a partir de los desechos de mora y de fresa, así como su uso en productos con alto valor agregado.

Palabras clave: desecho; antioxidante; minerales; vitamina C; conservación de alimentos; Fragaria ananassa; Rubus glaucus Benth. 
Aguirre, L.; Cubillos, L.; Tarazona-Díaz, M.; Rodriguez, L.: Potencial subproductos fresa y mora

\section{ABSTRACT}

Near of one third of the edible food that human being cultivate are loses, which are due to factors from the supply chain. Within these was highlighted strawberry (Fragaria ananassa) and blackberry (Rubus glaucus Benth), fruits that have demonstrated high antioxidant potential to which is related with prevention of some diseases. Therefore, with the aim to show the processing by-products' potential in the obtaining of interesting compounds, this study was focused on the characterization of their physicochemical parameters, color, antioxidant power and vitamin $\mathrm{C}$ in four preservation conditions for four weeks; these were: fresh by-product, refrigerated, conventional drying and lyophilization; also, mineral description was made for each sample. According to the results, it was proved that the samples had a lower solid soluble concentration, $\mathrm{pH}$ between 3.2 and 6.4, and stable color parameters during the storage. In addition, by-products with greater antioxidant power were lyophilizate blackberry seed and conventional drying blackberry sludge, which surpassed the strawberry's results in all the conditions conforming to FRAP and DPPH essays. However, all the samples presented low content of vitamin $C$, while they exhibited high content of potassium and iron. To sum up, above mentioned woke the interest on the minerals or antioxidant obtention from blackberry and strawberry by-products to which can be used in products with high value added.

Keywords: waste; antioxidant, minerals; vitamin C; food preservation; Fragaria ananassa; Rubus glaucus Benth.

\section{INTRODUCCIÓN}

La fresa (Fragaria ananassa) y la mora (Rubus glaucus Benth) son frutas cuya producción en Colombia superó las 60.000 y 100.000t, respectivamente, durante el 2016 (MinAgricultura, 2018). Dentro de sus aspectos nutricionales, ambas frutas tienen un alto potencial antioxidante $(17,0 \pm 1,3 \mu \mathrm{mol}$ Trolox/g, para fresa fresca variedad Maya, según ensayo FRAP (Capocasa et al. 2008) y hasta $6956 \pm 472 \mu \mathrm{mol}$ Trolox $/ 100 \mathrm{~g}$ de mora fresca, de acuerdo con el ensayo DPPH (Horvitz et al. 2017)mass loss, color, firmness, pH, total soluble solids, titratable acidity), siendo, a su vez, fuente de minerales $(235,15 \mathrm{mg} / 100 \mathrm{~g}$, de mora y $207,55 \mathrm{mg} / 100$, fresa) y vitamina C (21 mg ácido ascórbico/ $100 \mathrm{~g}$ de mora y 58,8mg ácido ascórbico/100g, fresa) (USDA, 2018), los cuales son necesarios en una dieta balanceada.

En los últimos años, uno de los factores que ha provocado el aumento en el consumo de frutas y de hortalizas es la prevención o el control de enfermedades (Figueira et al. 2016). En algunos casos, lo anterior puede ser explicado por la presencia de antioxidantes, los cuales, han demostrado propiedades terapéuticas contra enfermedades, como la diabetes tipo 2 (Johnson \& Gonzalez de Mejia, 2016), el infarto de miocardio (Cassidy et al. 2016) o los padecimientos neurodegenerativos, como el Alzheimer (Chang et al. 2013). Conforme a lo anterior, la identificación de dichos compuestos, a partir de los desechos de la agroindustria, podrían diversificar el uso de los coproductos del sector alimentario.
La industria de alimentos ocupa una importante posición económica y genera grandes volúmenes de residuos, en su mayoría biodegradables, los cuales, se producen a lo largo del proceso, en gran cantidad y no son aprovechados adecuadamente. Un informe de la Organización de las Naciones Unidas para la Alimentación y la Agricultura (FAO) determinó que, cerca de un tercio de las partes comestibles de los alimentos para el consumo humano, se pierde o se desperdicia, ya que, sin importar el nivel de industrialización de los países, dichas pérdidas se generan, en mayor o menor medida, desde la producción primaria hasta el consumo final en los hogares (FAO, 2012). Por consiguiente, existe un gran interés por dar valor agregado a los coproductos del beneficio agrícola, siendo fuentes de compuestos con potencial nutracéutico y antioxidante, tales como el resveratrol, la cumarina, $\beta$-caroteno entre otros (da Silva et al. 2014).

En este trabajo. se realizó la caracterización y la cuantificación de antioxidantes, parámetros fisicoquímicos (humedad, $\mathrm{pH},{ }^{\circ}$ Brix y color) y nutricionales (minerales y vitamina $\mathrm{C}$ ), para residuos de fresa y de mora, en diferentes métodos de conservación, con el fin de dar a conocer su potencial en la obtención de compuestos bioactivos, generar interés en su recuperación, diversificar el uso de los subproductos, propios del beneficio de dichas frutas y así, disminuir la pérdida y el desperdicio de los mismos, durante la cadena productiva.

\section{MATERIALES Y MÉTODOS}

Material vegetal. La fresa y la mora, se adquirieron en un mercado local y los residuos, se obtuvieron tras despulpar la fruta en una máquina despulpadora (DE-101, Caval, Bogotá, Colombia), con una malla de $0,75 \mathrm{~mm}$; posteriormente, $2 \mathrm{~kg}$ de mezcla de residuos, corteza, semilla y lodo, se congelaron hasta su pretratamiento $\left(-18 \pm 1^{\circ} \mathrm{C}\right)$.

Preparación de la muestra y diseño experimental. El material vegetal se descongeló en un horno microondas (700W), durante 10 minutos, alcanzando una temperatura de $4^{\circ} \mathrm{C}$, de acuerdo con lo descrito por Holzwarth et al. (2012respectively, and subsequently thawed at various temperatures $\left(+4,+20\right.$ and $+37^{\circ} \mathrm{C}$, respectively). Posteriormente, las muestras fueron distribuidas, con el fin de disponerlas en cuatro condiciones de conservación, las cuales, fueron: fresco $\left(21,6 \pm 1^{\circ} \mathrm{C}\right.$ y $35 \pm 4 \%$, de humedad relativa), refrigerado $\left(4 \pm 1^{\circ} \mathrm{C}\right.$ y $26 \pm 4 \%$, de humedad relativa), secado convencional $\left(37^{\circ} \mathrm{C}\right.$ durante $24 \mathrm{~h}$, en estufa de secado, MLW Coimsa, USA) y liofilizado (fase de congelación a $-34^{\circ} \mathrm{C}$, durante $24 \mathrm{~h}$ y sublimación a $22^{\circ} \mathrm{C}, 4300 \mathrm{~Pa}$, durante 8 días, en liofilizador Drycol, Bogotá, Colombia). Previo al análisis, las muestras sometidas a deshidratación fueron molidas en un molino de palas (retsch sk 100, Hann, Alemania), usando un tamiz de 0,5mm de diámetro. Por último, las muestras fueron empacadas al vacío (multivac chamber machine C200, Bremen, Alemania) y almacenadas a $21,6 \pm 1^{\circ} \mathrm{C}$ y $35 \pm 4 \%$, de humedad relativa.

El diseño experimental consistió en evaluar, durante 4 semanas, el comportamiento de los parámetros fisicoquímicos, antioxidantes y vitamina $\mathrm{C}$, teniendo 4 variables correspondientes a los pretratamientos, como fresco, refrigerado, secado, liofilización. Para el aná- 
lisis estadístico, se realizó un análisis de varianza (ANOVA, $\mathrm{p}<0,05$; Statgraphic Plus, 5.1, Manugistic Inc, Rockville, MD, EE.UU).

Para la determinación de antioxidantes y vitamina $C$, se realizaron extractos en relación (1:40), con una solución de metanol al 80\%, los cuales, se centrifugaron a 6000 RPM, durante $15 \mathrm{~min}$, en una centrífuga (Rotofix 32 A KASAI Hettich zentrifugen, Canadá). Para los análisis fisicoquímicos, se realizaron extractos (1:5) con agua destilada, excepto para el análisis de color y humedad, medidos directamente sobre la muestra.

Humedad. 0,5g de muestra fueron colocados en una balanza de humedad (Mettler toledo HG43-s Halogen, Switzerland, Suiza). Después del tiempo de medición, el equipo arrojó el contenido de agua de la muestra expresado en porcentaje (\%).

Potencial de hidrógeno $(\mathrm{pH})$. Se utilizó un Potenciómetro S20 SevenEasy, (Mettler Toledo, Suiza), de acuerdo con el método AOC 943.02 (AOAC, 1995).

Sólidos solubles totales ( ${ }^{\circ}$ Brix). Se evaluó por refractometría, utilizando un refractómetro digital (ATAGO PAL-alpha, USA) previamente calibrado con agua destilada. El resultado fue expresado como grados Brix ( ${ }^{\circ}$ Brix) y corregidos (ICONTEC, 1997), de acuerdo con la ecuación 1.

SSTcor $=0,194 * A+S S T$

ecuación 1

Donde, SSTcor: sólidos solubles totales corregido ( $\left.{ }^{\circ} \mathrm{Brix}\right), \mathrm{A}$ : porcentaje de ácido, SST: sólidos solubles totales ( ${ }^{\circ}$ Brix).

Color. Para la evaluación del color, se utilizó un Colorímetro CIE. Lab (Konica Minolta CR-400, Japón), con lecturas en términos de $L^{*}$, a* y b*, empleando una placa de $\mathrm{BaSO}_{4}$ estándar (X 79,80, Y 84,97, Z 90,74), para la calibración del instrumento. Las medidas de color de las muestras, se llevaron a cabo en la superficie de ésta, donde se calculó croma (Ecuación 2), índice de color (Ecuación 3), ángulo Hue (Ecuación 4) y diferencia de color (Ecuación 5) (Sahin \& Summu, 2006).

$$
\begin{aligned}
& C^{*}=\sqrt{a^{*^{2}+b^{* 2}}} \\
& I C=\frac{a^{*} 1000}{L^{*} b^{*}}
\end{aligned}
$$
ecuación 2 ecuación 3

${ }^{\circ} \mathrm{Hue}=\arctan \left(\frac{\mathrm{b}^{*}}{\mathrm{a}^{*}}\right)$

$$
\Delta \mathrm{E}^{*}=\left[\left(\Delta \mathrm{L}^{*}\right)^{2}+\left(\Delta \mathrm{a}^{*}\right)^{2}+\left(\Delta \mathrm{b}^{*}\right)^{2}\right.
$$

ecuación 5

Dónde, L*, luminosidad; $a^{*}$, posición entre rojo y verde; $b^{*}$, posición entre amarillo y azul; C, croma; IC, índice de color; ${ }^{\circ}$ Hue, ángulo de tono; $\Delta \mathrm{E}^{*}$, diferencia de color; $\Delta \mathrm{L}^{*}$, diferencia de luminosidad;
$\Delta \mathrm{a}^{*}$, diferencia en la posición entre rojo y verde; $\Delta \mathrm{b}^{*}$, diferencia en la posición entre amarillo y azul.

Determinación del contenido de minerales. Se basó en la digestión de las muestras por triplicado, con ácido nítrico, en un recipiente cerrado, donde se controlaron variables de temperatura y de tiempo, mediante un digestor microondas. La metodología consistió en pesar $0,3 \mathrm{~g}$ de muestra seca directamente, dentro de un tubo de digestión y adicionar 9mL de HNO3 (65\%), siendo este tapado de inmediato. El programa de digestión consistió en una rampa de $180^{\circ} \mathrm{C} / 5 \mathrm{~min}$ y, luego, de $180^{\circ} \mathrm{C} / 10 \mathrm{~min}$, con una potencia de 700W. Los tubos, se enfriaron y las muestras se trasladaron a un matraz aforado de $100 \mathrm{~mL}$, con una solución de HNO3 (1\%), con agua Milli-Q, determinando, así, manganeso (Mn), boro (B), zinc $(\mathrm{Zn})$, calcio $(\mathrm{Ca})$, fósforo $(\mathrm{P})$, potasio $(\mathrm{K})$, hierro $(\mathrm{Fe})$, sodio $(\mathrm{Na})$ y magnesio $(\mathrm{Mg})$, por espectrometría de masas por plasma de acoplamiento inductivo (ICP-MS, 7500ce, Agilent Technologies, Manchester, U.K.) (EPA, 2007).

Determinación del contenido de vitamina C. Esta técnica tiene como fundamento el ácido ascórbico, tratado con 2-nitroanilina diazotada, pasando a 2-nitrofenilhidrazida del ácido, el cual, en presencia de un exceso de $\mathrm{NaOH}$, forma una sal sódica de color rojovioleta, que tiene un máximo de absorción de $540 \mathrm{~nm}$. El ensayo, se realizó como lo describe Bernal de Ramírez (1998) por triplicado, que consistió en tomar una alícuota de $10 \mu \mathrm{L}$ de 2 - nitroanilina $0,16 \%$ y $20 \mu \mathrm{L}$ de nitrito de sodio $0,08 \%$, recién preparado; luego, se adicionó $380 \mu \mathrm{L}$ de etanol $96 \%, 50 \mu \mathrm{L}$ de la muestra (extracto) y $50 \mu \mathrm{L}$ de ácido oxálico $0,15 \%$; se mezcló y se dejó en reposo 5 minutos; posteriormente, se adicionó $120 \mu \mathrm{L}$ de $\mathrm{NaOH} 10 \%$ y $380 \mu \mathrm{L}$ de agua destilada. Finalmente, la mezcla se leyó en el espectrofotómetro (Thermo Scientific Evolution 300, EE.UU), a una absorbancia de 540nm y se comparó con una curva de calibración de ácido ascórbico $\left(\mathrm{R}^{2}>0.99\right)$. Para el blanco, se tomó una alícuota de $50 \mu \mathrm{L}$ de ácido oxálico, como muestra durante el experimento.

Determinación de antioxidantes por el método de FRAP. Este método, se basa en la reducción del ion férrico $\left(\mathrm{Fe}^{3+}\right)$ a ion ferroso $\left(\mathrm{Fe}^{2+}\right)$, en condiciones ácidas; se determinó, de acuerdo con el método descrito por Benzie \& Strain (1996) por triplicado. 940 $\mathrm{L}$ de reactivo FRAP (compuesto por buffer acetato de sodio 0,3M, con $\mathrm{pH}$ 3,6, TPTZ 0,01M y cloruro férrico 0,02M (10:1:1)), fueron mezclados con $30 \mu \mathrm{L}$ de etanol-agua $(80: 20)$ y $30 \mu \mathrm{L}$ de extracto en un tubo Eppendorf. Esta disolución, se incubó a $37^{\circ} \mathrm{C}$, durante $1 \mathrm{~h}$, en ausencia de luz, tras ello, se homogeneizó en vortex. Posteriormente, se leyó la absorbancia a 593nm, en el espectrofotómetro (Espectrofotómetro evolution 300 thermo scientific, Matlock, Inglaterra) y se comparó con una curva de calibración de Trolox $\left(\mathrm{R}^{2}>0,99\right)$. Para el blanco, se tomó una alícuota de $940 \mu \mathrm{L}$ de FRAP y $60 \mu \mathrm{L}$ de metanol-agua (80:20).

Determinación de antioxidantes por el método de DPPH. Se basa en el principio de aceptación de un hidrógeno $(\mathrm{H})$ en el átomo de la molécula captadora DPPH, dando la reducción de DPPH a $\mathrm{DPPH}_{2}$, el cual, se determinó de acuerdo con el método descrito 
por Brand-Williams et al. (1995) por triplicado; para ello, se tomó una alicuota de $200 \mu \mathrm{L}$ de reactivo DPPH, $800 \mu \mathrm{L}$ de metanol-agua (80:20) y $50 \mu \mathrm{L}$ del extracto; luego, se incubó a $37^{\circ} \mathrm{C}$, durante una hora y en ausencia de luz se homogenizó en vortex; posteriormente, se leyó la absorbancia en el espectofotómetro a 515nm (Thermo Scientific Evolution 300,EEUU) y se comparó con una curva de calibración de Trolox $\left(\mathrm{R}^{2}>0.99\right)$. Para el blanco, se tomó una alícuota de 1000 $\mu \mathrm{L}$ de metanol-agua (80:20).

\section{RESULTADOS Y DISCUSIÓN}

Parámetros fisicoquímicos. El máximo valor de humedad alcanzado fue para los residuos de fresa, que fue de $88 \%$, en fresco y refrigerado (Tabla 1), similares al contenido de humedad reportado para mora y para frambuesa $(86,13 \pm 0,12 \%$ y $85,84 \pm 1,66 \%$, respectivamente) (Yang \& Choi, 2016).

Los residuos de mora y de fresa presentaron $\mathrm{pH}$ entre 3,2 (lodo de mora liofilizado) y 4,7 (semilla de mora liofilizada), durante la semana cero (Tabla 1), siendo comparables con los valores determinados por Yang \& Choi (2016), para frambuesa silvestre (3,39 $\pm 0,11)$, mora $(3,49 \pm 0,10)$ y morera $(4,73 \pm 0,11)$; a su vez, son similares a lo obtenido por de Souza et al. (2014), para fresa $(3,73 \pm 0,01)$ y arándanos $(3,64 \pm 0,05)$. Durante las semanas de almacenamiento, las muestras secas mostraron mayor estabilidad en el pH en comparación con los residuos frescos y refrigerados; lo anterior, puede ser explicado por la reducción de humedad en las muestras, que permitió la inhibición de los procesos de deterioro, disminuyendo la actividad enzimática y los cambios químicos relacionados.

Las muestras secas presentaron el mayor contenido de sólidos solubles totales (Tabla 1), como es el caso de los residuos de fresa liofilizada (entre $10-12^{\circ}$ Brix), similares a lo reportado en fresa $\left(10,50 \pm 0,50^{\circ}\right.$ Brix), frambuesa roja $\left(10,33 \pm 0,58^{\circ}\right.$ Brix) (de Souza et al. 2014) y arándano rojo $\left(9,93 \pm 0,05^{\circ}\right.$ Brix) (Yang \& Choi, 2016). Los sólidos solubles en las muestras de lodo y semilla de mora liofilizada $\left(5,4\right.$ y $6,3^{\circ} \mathrm{Brix}$, respectivamente), son similares a lo obtenido por Van de Velde et al. (2016), para mora fresca de variedad 'Jumbo' (5,5 $\pm 1,0^{\circ}$ Brix), 'Blacksatin' $\left(7,0 \pm 1,0^{\circ}\right.$ Brix) y 'Dirksen' $\left(6,9 \pm 0,6^{\circ}\right.$ Brix $)$; no obstante, la semilla de mora liofilizada sufrió

Tabla 1. Parámetros fisicoquímicos de los residuos de mora y de fresa.

\begin{tabular}{|c|c|c|c|c|c|c|c|c|c|c|c|c|}
\hline \multicolumn{13}{|c|}{$\%$ Humedad } \\
\hline \multirow{2}{*}{$\mathbf{T}$} & \multicolumn{3}{|c|}{ Fresco } & \multicolumn{3}{|c|}{ Refrigerado } & \multicolumn{3}{|c|}{ Secado C. } & \multicolumn{3}{|c|}{ Liofilizado } \\
\hline & SM & LM & $\mathbf{F}$ & SM & $\mathbf{L M}$ & $\mathbf{F}$ & SM & LM & $\mathbf{F}$ & SM & LM & F \\
\hline 0 & 72 & 81 & 82 & 75 & 81 & 89 & 6.1 & 5,9 & 10 & 4,9 & 5,3 & 13 \\
\hline 1 & 74 & 82 & 88 & 75 & 82 & 86 & 5,3 & 6,1 & 12 & 5 & 4,9 & 12 \\
\hline 2 & 75 & 83 & 88 & 74 & 82 & 85 & 5,4 & 5,9 & 13 & 4,6 & 5,4 & 12 \\
\hline 3 & - & - & - & 75 & 83 & 88 & 5,6 & 6,3 & 14 & 4,7 & 6 & 10 \\
\hline 4 & - & - & - & 72 & 82 & 84 & 5 & 6,3 & 13 & 6 & 5,7 & 12 \\
\hline \multicolumn{13}{|c|}{$\mathrm{pH}$} \\
\hline \multirow{2}{*}{$\mathbf{T}$} & \multicolumn{3}{|c|}{ Fresco } & \multicolumn{3}{|c|}{ Refrigerado } & \multicolumn{3}{|c|}{ Secado C. } & \multicolumn{3}{|c|}{ Liofilizado } \\
\hline & SM & $\mathbf{L M}$ & $\mathbf{F}$ & SM & $\mathbf{L M}$ & $F$ & SM & LM & $\mathbf{F}$ & SM & LM & $\mathbf{F}$ \\
\hline 0 & 4,3 & 3,4 & 3,6 & 4,7 & 3,4 & 3,6 & 4,1 & 3,2 & 3,8 & 3,6 & 3,2 & 3,5 \\
\hline 1 & 5.8 & 3,8 & 3,4 & 5,4 & 3,3 & 3,5 & 4,5 & 3,3 & 3,8 & 3,8 & 3,3 & 3,8 \\
\hline 2 & 6.4 & 4,1 & 3,5 & 5,4 & 3,42 & 3,3 & 4 & 3,2 & 3,7 & 3,9 & 3,4 & 3,7 \\
\hline 3 & - & - & - & 5,4 & 3,5 & 3,4 & 4,2 & 3,3 & 3,8 & 3,8 & 3,4 & 3,7 \\
\hline 4 & - & - & - & 5,7 & 3,4 & 3,2 & 4,4 & 3,3 & 3,8 & 4 & 3,5 & 3,9 \\
\hline \multicolumn{13}{|c|}{ Sólidos solubles ( ${ }^{\circ} \mathrm{Brix}$ ) } \\
\hline \multirow{2}{*}{$\mathbf{T}$} & \multicolumn{3}{|c|}{ Fresco } & \multicolumn{3}{|c|}{ Refrigerado } & \multicolumn{3}{|c|}{ Secado C. } & \multicolumn{3}{|c|}{ Liofilizado } \\
\hline & SM & $\mathbf{L M}$ & $\mathbf{F}$ & SM & $\mathbf{L M}$ & $F$ & SM & LM & $\mathbf{F}$ & SM & $\mathbf{L M}$ & $\mathrm{F}$ \\
\hline 0 & 0,3 & 3,6 & 0,3 & 0,9 & 0,5 & 1 & 3 & 4 & 10 & 6,3 & 5,4 & 12 \\
\hline 1 & 0,3 & 3,87 & 0,4 & 0,5 & 1 & 1,1 & 3,4 & 3,2 & 12 & 3,3 & 3,4 & 11 \\
\hline 2 & 0,5 & 4,04 & 0,3 & 0,5 & 0,5 & 0,6 & 2,7 & 4,7 & 8,1 & 2,9 & 3 & 11 \\
\hline 3 & - & - & - & 0,2 & 0,6 & 0,8 & 3,4 & 4 & 9,8 & 4,7 & 3,4 & 10 \\
\hline 4 & - & - & - & 0,4 & 0,7 & 0,8 & 2,6 & 3,4 & 12 & 0,7 & 3,5 & 11 \\
\hline
\end{tabular}

$\mathrm{SM}=$ Semilla de mora, $\mathrm{LM}=$ Lodo de mora, $\mathrm{F}=$ Residuo de fresa. 
una disminución en el contenido de sólidos solubles, especialmente, durante la semana 4, que puede estar relacionada con el aumento en la humedad, dada a causa de las condiciones de almacenamiento.

Color. De acuerdo con las figuras $1 \mathrm{~A}$ y $2 \mathrm{~A}$, el croma se mantuvo estable en todas las condiciones de experimentación durante el tiempo de almacenamiento; dicho parámetro estuvo entre 9,33 (semilla de mora fresca, durante la primera semana) y 24,3 (residuo de fresa liofilizado, en la tercera semana), siendo las muestras secas, las que presentaron mayor saturación con respecto a los productos frescos y refrigerados. Por otro lado, el ángulo Hue no presentó ningún cambio con los pretratamientos o la materia prima, obteniendo para todas las condiciones, un ${ }^{\circ} \mathrm{Hue}$ de $1,54 \pm 0,01$. Por consiguiente, la diferencia de color (figura $1 \mathrm{~B}$ y $2 \mathrm{~B}$ ), con respecto al estándar $(\mathrm{L}=$ $71,54, a^{*}=12,87 \mathrm{y} \mathrm{b}=6,76$ ), estuvo entre 1,51 (residuo de fresa fresca, durante la semana 0) y 11,44 (residuo de fresa liofilizado, en la cuarta semana). De acuerdo con Obón et al. (2009), las diferencias de color empiezan a ser evidentes cuando el parámetro es superior a 5 unidades, siendo el residuo de fresa liofilizado la muestra que más difiere respecto al estándar de medición. Lo anterior, se puede explicar, debido al aumento en la concentración de pigmentos, tales como antocianinas en las muestras secas, generando colores más intensos a comparación de los productos frescos, cuya diferencia con el estándar es menor.

Según la figura 1C y 2C, el índice de color osciló entre 15,27 y 43,63, representando desde el naranja intenso hasta el rojo profundo, respectivamente (Vignoni et al. 2006); sin embargo, es de resaltar que compuestos, como las antocianinas, se ven afectados por la temperatura (Liu et al. 2018), generando cambios de color dentro de la matriz de las materias primas, sometidas a secado convencional. Por ende, lo anterior puede explicar la variabilidad en el índice de color del lodo de mora seco por métodos convencionales, cuyos resultados no muestran un comportamiento definido durante el almacenamiento.

Determinación del contenido de minerales. De acuerdo con la tabla 2, las semillas de mora son el coproducto con mayor conteni-
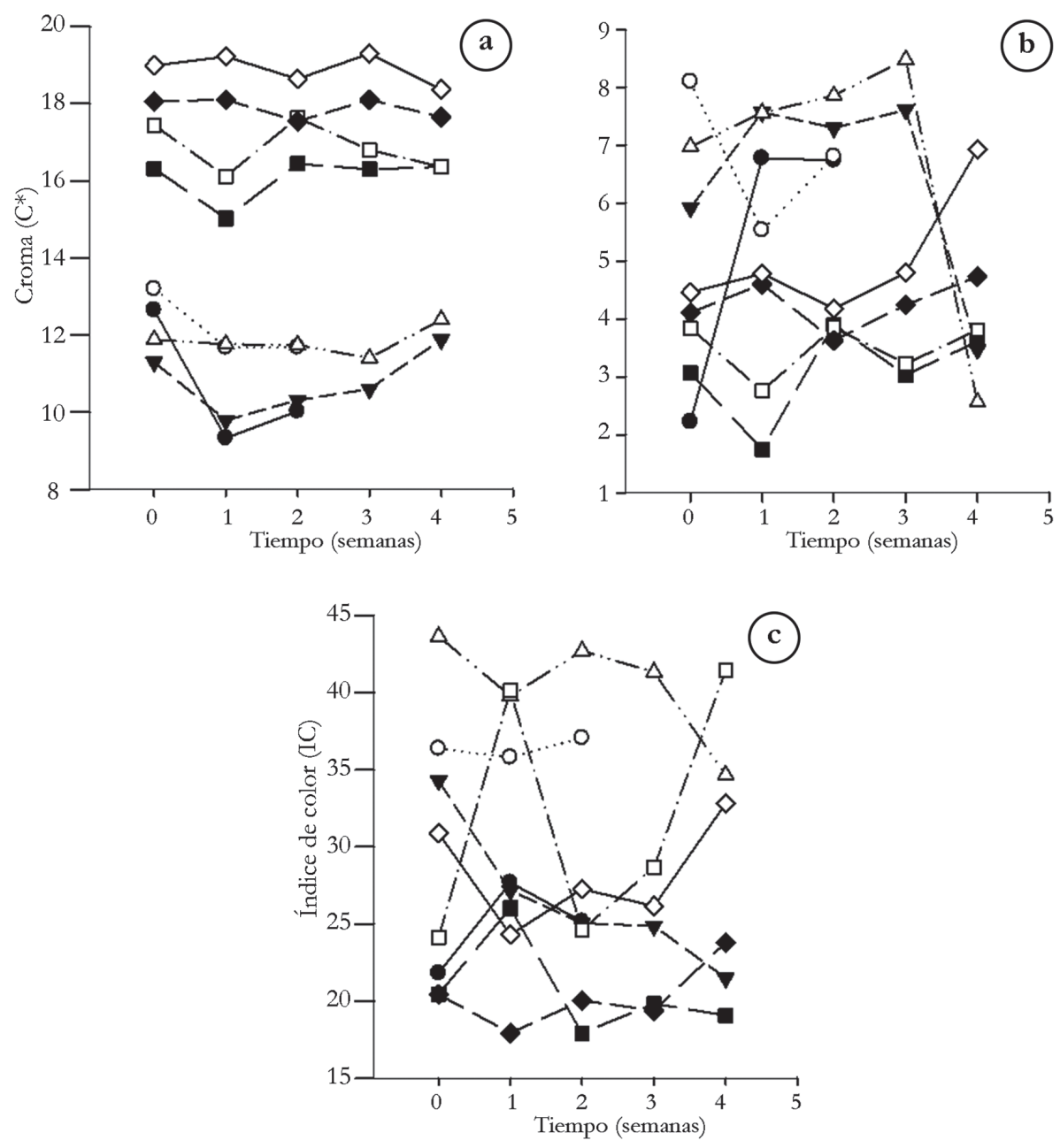

Figura 1. Parámetros de color para los residuos de mora ( semilla de mora fresca, o lodo de mora fresco, $\boldsymbol{\nabla}$ semilla de mora refrigerada, $\Delta$ lodo de mora refrigerado, $\mathbf{a}$ semilla de mora por secado convencional, $\square$ lodo de mora por secado convencional, $\boldsymbol{}$ semilla de mora liofilizada, $\diamond$ lodo de mora liofilizado): a. croma; b. diferencia de color; c. índice de color. 

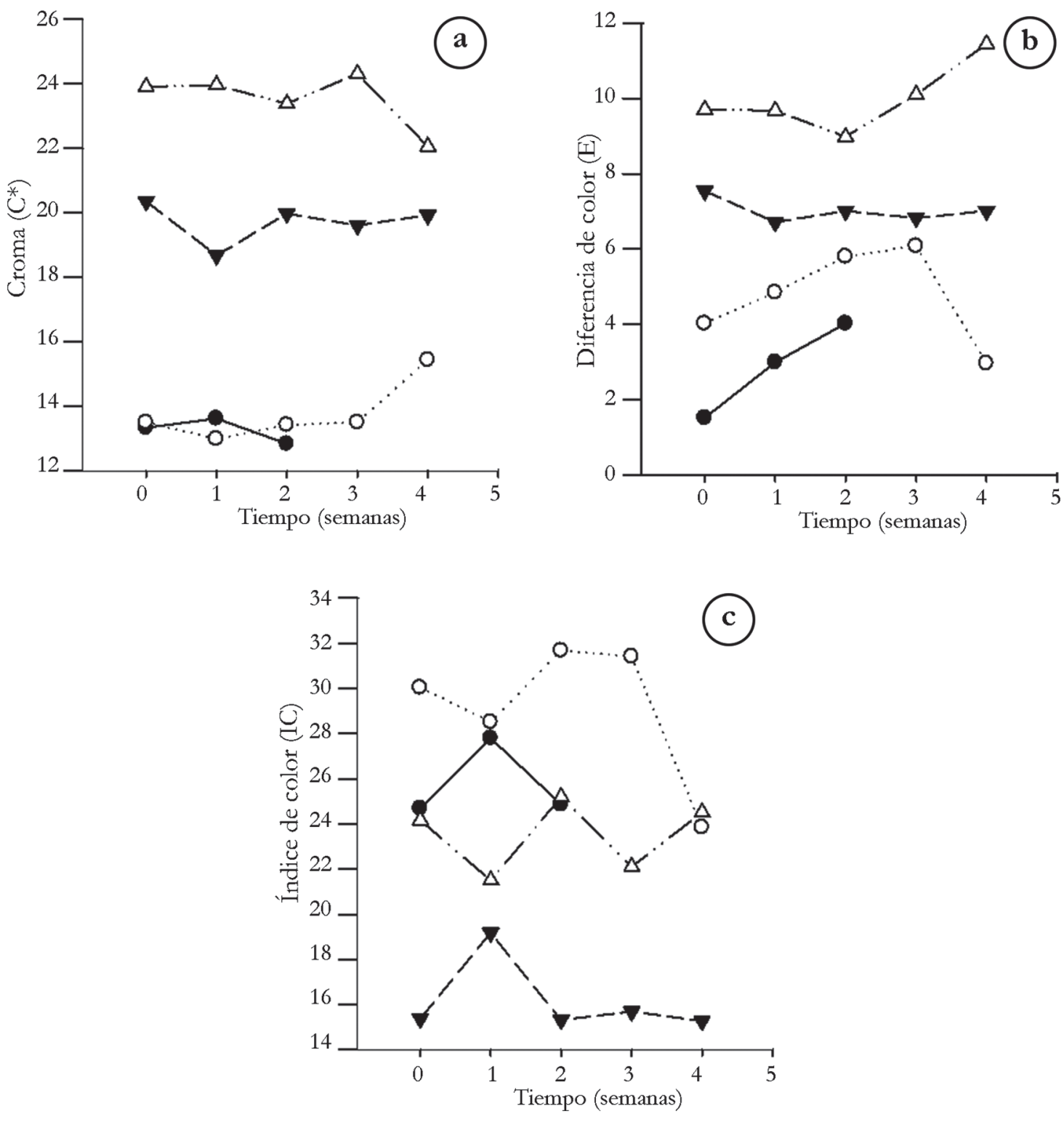

Figura 2. Parámetros de color para los residuos de fresa ( $\bullet$ fresco, $\bigcirc$ refrigerado, $\nabla$ secado convencional, $\Delta$ liofilizado): a. croma; b. diferencia de color; c. índice de color.

do en minerales y en nitrógeno (de acuerdo con prueba de Tukey $(\mathrm{p}<0.05)$; a excepción del potasio, cuya concentración fue superior en los residuos de fresa $(1,47 \pm 0,06 \mathrm{~g} / 100 \mathrm{~g}$, de muestra seca en base seca); por consiguiente, el coproducto con menor cantidad de minerales fue el lodo de mora.

El elemento mayor con más alta presencia en los subproductos de fresa y de mora fue el potasio, superando lo reportado para semillas de fresa desengrasadas (0,4,8g de K/100g d.m) (Grzelak-Błaszczyk et al. 2017) y para subproductos de manzana $(0,71 \mathrm{~g} / 100 \mathrm{~g})$; sin embargo, se ha reportado mayor cantidad de potasio en subproductos de papa $(3,73 \mathrm{~g} / 100 \mathrm{~g})$, pepino $(4,61 \mathrm{~g} / 100 \mathrm{~g})$, melón $(3,53 \mathrm{~g} / 100 \mathrm{~g})$ y sandía (4,77 g/100 g) (Tarazona-Díaz \& Aguayo, 2013). Adicionalmente, el elemento menor predominante en los coproductos estudiados es el hierro, superior a lo reportado para subproductos de manzana $(33,8 \mathrm{mg} / \mathrm{kg})$, pepino $(81 \mathrm{mg} / \mathrm{kg})$, melón $(66,6 \mathrm{mg} / \mathrm{kg})$ y sandía (59,1 mg/kg) (Tarazona-Díaz \& Aguayo, 2013). Por otro lado, los subproductos estudiados tienen una baja presencia en sodio y cobre (ambos con menos de $1 \mathrm{mg} / 100 \mathrm{~g}$, en base seca). Conforme a lo anterior, los coproductos del beneficio de la mora y la fresa tienen potencial para su utilización en productos que requieran el enriquecimiento de minerales, especialmente, de potasio y de hierro.

Poder antioxidante y contenido de vitamina C. Los coproductos que tienen mayor poder antioxidante, según ensayo FRAP, fueron la semilla de mora y el residuo de fresa, ambos liofilizados $(21,79 \pm 0,12$ y 16,50 $\pm 0,01 \mathrm{mg}$ Trolox eq/g, respectivamente) (Figuras 3a y 4a), mientras que por DPPH, el lodo de mora y el residuo de fresa, 
ambos por secado convencional $(13,28 \pm 0,017 \mathrm{mg}$ y12,73 $\pm 0,01 \mathrm{mg}$ Trolox eq/g), fueron los que presentaron los mayores resultados (Figura 3b y 4b). La disminución del contenido de agua, debida a los tratamientos, pudo ser la causante de la alta concentración de antioxidantes en los subproductos, siendo mayor a lo reportado para grosellas, almacenadas a 25 y $4^{\circ} \mathrm{C}(1,85$ y $1,75 \mathrm{~mm}$ Trolox eq/ $100 \mathrm{~g}$ FW, respectivamente, según ensayo DPPH) (Piljac-Žegarac \& Šamec, 2011), al extracto de fresa tipo Alba $(22,85 \pm 0,39$ y $7,71 \pm 0,32 \mu \mathrm{mol}$

Tabla 2. Contenido de minerales en residuos de mora y de fresa (media \pm desviación estándar $n=3$ ).

\begin{tabular}{|l|l|l|l|}
\hline Parámetro & Semilla de mora & Lodo de mora & Residuo de fresa \\
\hline Nitrogeno $^{1}$ & $2,31 \pm 0,03^{\mathrm{a}}$ & $1,54 \pm 0,05^{\mathrm{b}}$ & $1,49 \pm 0,05^{\mathrm{b}}$ \\
\hline Fosforo $^{1}$ & $0,178 \pm 0,04^{*}$ & $0,082 \pm 0,002^{*}$ & $0,243 \pm 0,004^{*}$ \\
\hline Potasio $^{1}$ & $0,816 \pm 0,004^{\mathrm{b}}$ & $0,61 \pm 0,01^{\mathrm{c}}$ & $1,47 \pm 0,06^{\mathrm{a}}$ \\
\hline Calcio $^{1}$ & $0,55 \pm 0,01^{\mathrm{a}}$ & $0,175 \pm 0,002^{\mathrm{c}}$ & $0,344 \pm 0,004^{\mathrm{b}}$ \\
\hline Sodio $^{1}$ & $0,0063 \pm 0,0012^{\mathrm{a}}$ & $0,0063 \pm 0,0012^{\mathrm{a}}$ & $0,0063 \pm 0,0012^{\mathrm{a}}$ \\
\hline Magnesio $^{1}$ & $0,159 \pm 0,001^{*}$ & $0,060 \pm 0,001^{*}$ & $0,175 \pm 0,001^{*}$ \\
\hline Azufre $^{1}$ & $0,152 \pm 0,002^{\mathrm{a}}$ & $0,091 \pm 0,002^{\mathrm{b}}$ & $0,086 \pm 0,01^{\mathrm{b}}$ \\
\hline Hierro $^{2}$ & $173,67 \pm 2,89^{\mathrm{a}}$ & $116,0 \pm 1,73^{\mathrm{b}}$ & $175,67 \pm 3,21^{\mathrm{a}}$ \\
\hline Manganeso $^{2}$ & $86,3 \pm 2,2^{\mathrm{a}}$ & $43,9 \pm 2,8^{\mathrm{c}}$ & $78,69 \pm 0,72^{\mathrm{b}}$ \\
\hline Zinc $^{2}$ & $35,7 \pm 1,4^{\mathrm{a}}$ & $19,8 \pm 2,2^{\mathrm{b}}$ & $19,4 \pm 1,0^{\mathrm{b}}$ \\
\hline Cobre $^{2}$ & $9,62 \pm 0,23^{\mathrm{a}}$ & $5,63 \pm 0,12^{\mathrm{c}}$ & $6,97 \pm 0,25^{\mathrm{b}}$ \\
\hline Boro $^{2}$ & $55,68 \pm 1,62^{\mathrm{a}}$ & $28,09 \pm 0,67^{\mathrm{b}}$ & $21,55 \pm 0,53^{\mathrm{c}}$ \\
\hline
\end{tabular}

Letras iguales en la misma fila significa que no hay diferencias significativas, basadas en la prueba de Tukey $(\mathrm{p}<0.05)$. *No es posible determinar diferencias significativas, basado en la prueba de normalidad Shapiro-Wilk $(\mathrm{p}<0.05)$. ${ }^{1}$ Parámetro expresado como g/100g muestra en base seca. ${ }^{2}$ Parámetro expresado como $\mathrm{mg} / \mathrm{kg}$ muestra en base seca.

Trolox eq/g FW, de acuerdo con ensayo FRAP y DPPH) (Gasparrini et al. 2017) y a la pulpa de mora 'Brazos' y 'Tupy' (64,63 $\pm 3,08$

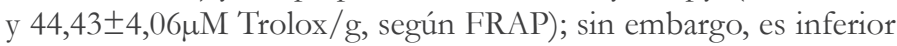
a lo determinado en corteza de sandía variedad Motril y Boston (0,062mg ácido ascórbico equivalente, según DPPH) (Tarazona-Díaz et al. 2010).

Lo anterior demuestra que los residuos de fresa y mora son fuente potencial para la obtención de compuestos con alto poder antioxidante.

Los subproductos tienen un bajo contenido de Vitamina C (Figura 3C y 4C), siendo el residuo de fresa, tratado por secado convencio- nal, el subproducto con mayor cantidad de ácido ascórbico $\left(7,1 * 10^{-3}\right.$ $\pm 7,4 * 10^{-4} \mathrm{mg}$ ácido ascórbico/L), inferior a lo reportado para limón sin corteza (53,0mg ácido ascórbico/100g) y guayaba (228,3mg ácido ascórbico/100g) (USDA, 2018), frutos que se destacan dentro de la dieta por su contenido en vitamina C. Asimismo, los resultados del presente estudio son inferiores a lo determinado para productos con bajo contenido en vitamina $\mathrm{C}$, como el Kiwano $(0,925 \mathrm{mg} / 100 \mathrm{~g})$ o la granada $(1,56 \mathrm{mg} / 100 \mathrm{~g})$ (Valente et al. 2011). Tal concentración de vitamina $C$ en los subproductos, se puede deber a que, durante el procesamiento de la fresa y la mora, cabe la posibilidad de que, tales materias primas, hayan sido sometidas a altas temperaturas, degradando la vitamina C, debido a su carácter termolábil (Athmaselvi et al. 2017), lo que hace que no tengan el potencial para la obtención de dicho compuesto. 

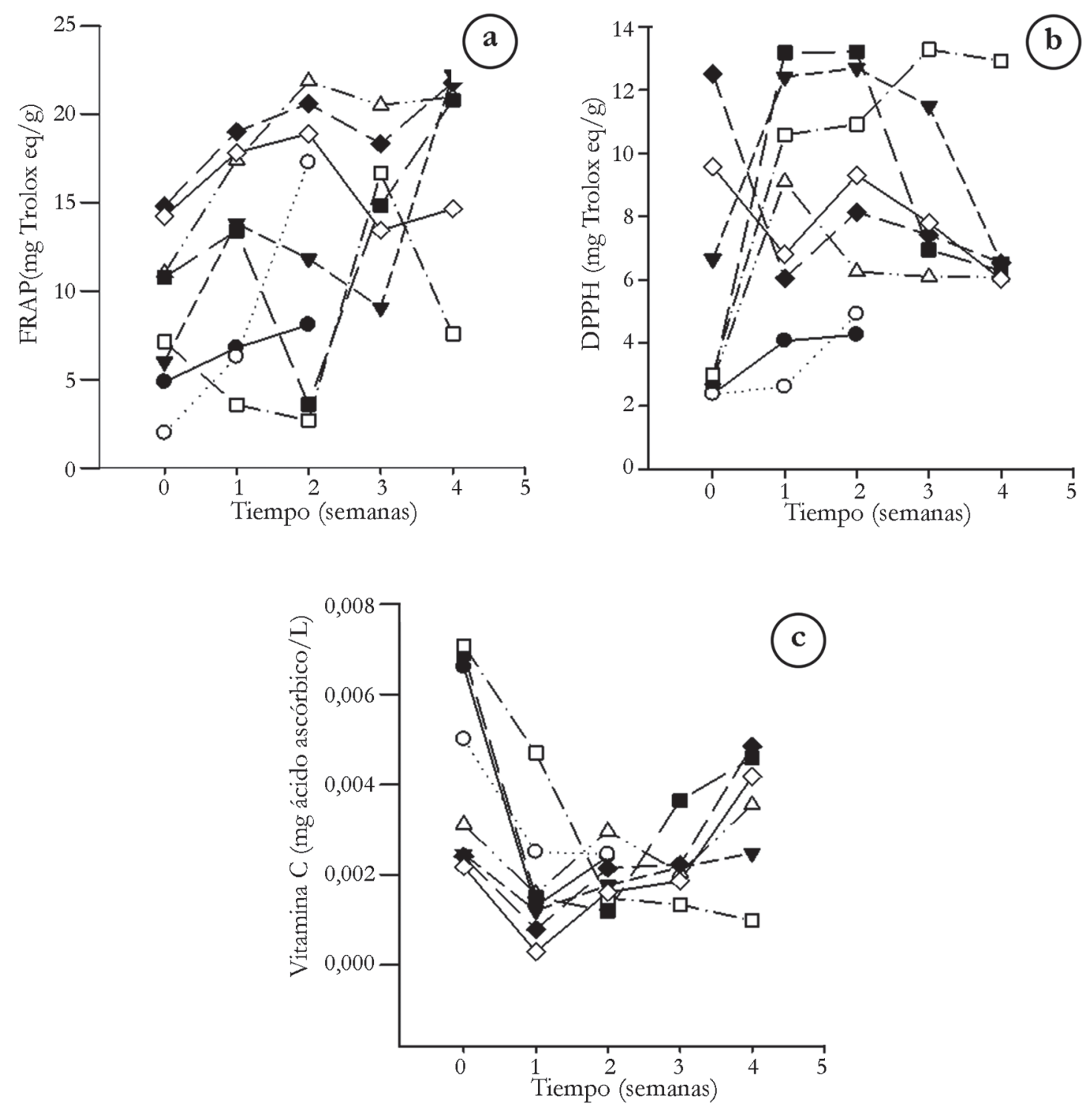

Figura 3. Poder antioxidante y contenido de vitamina $C$ para los residuos de mora $(\bullet$ semilla de mora fresca, $\circ$ lodo de mora fresco, $\nabla$ semilla de mora refrigerada, $\Delta$ lodo de mora refrigerado, $\mathbf{a}$ semilla de mora por secado convencional, $\square$ lodo de mora por secado convencional, semilla de mora liofilizada, $\diamond$ lodo de mora liofilizado): a. FRAP; b. DPPH; c. vitamina C. 

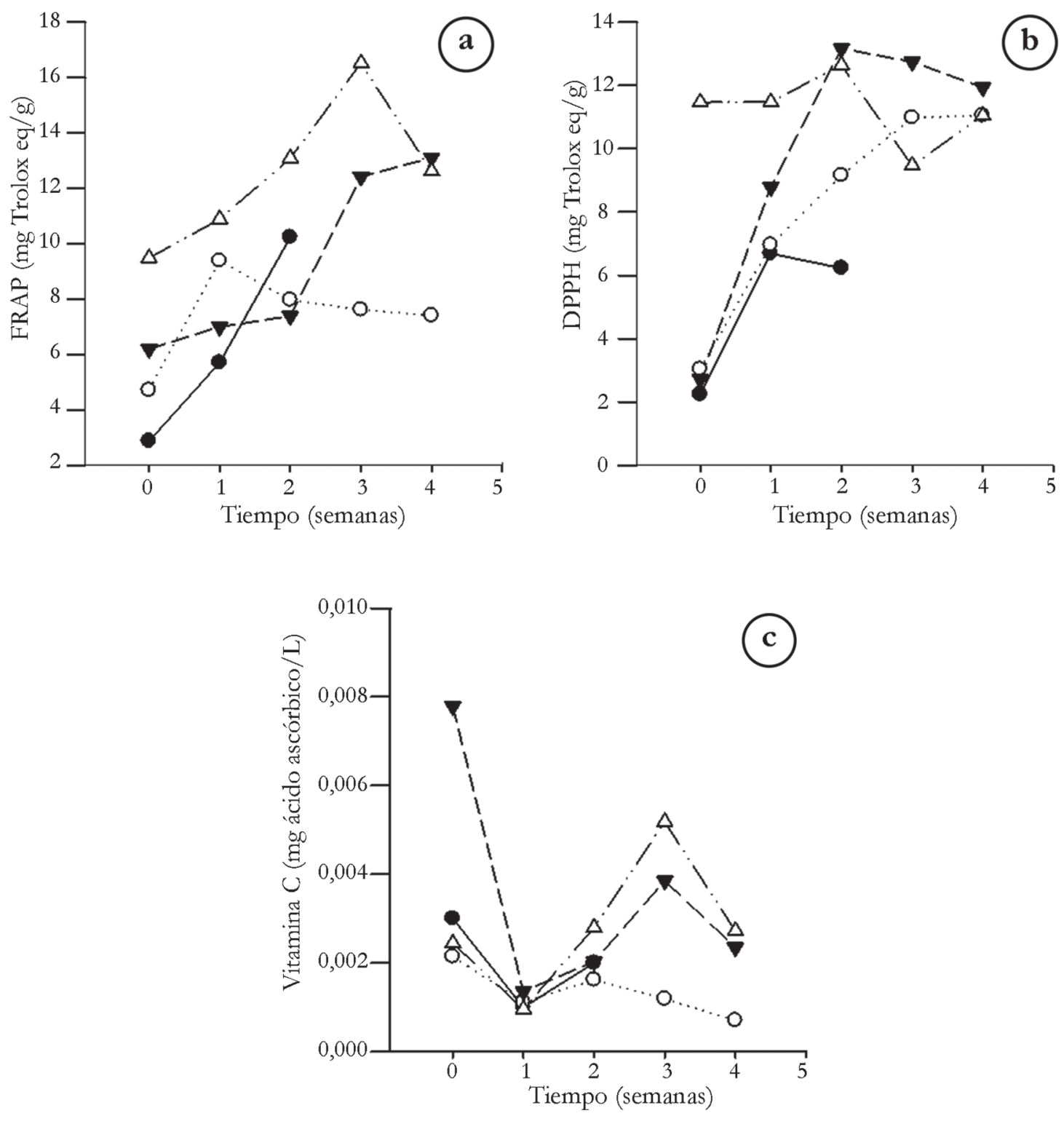

Figura 4. Poder antioxidante y contenido de vitamina $C$ para los residuos de fresa $(\bullet$ fresco, $\bigcirc$ refrigerado, $\nabla$ secado convencional, $\Delta$ liofilizado) a. FRAP; b. DPPH; c. vitamina C. 
Conflictos de intereses: El manuscrito fue preparado y revisado con la participación de todos los autores, quienes declaramos que no existe conflicto de intereses que ponga en riesgo la validez de los resultados presentados. Financiación: Este estudio fue financiado por la Universidad Jorge Tadeo Lozano.

\section{REFERENCIAS}

1. AOAC INTERNATIONAL. 1995. 943.02. pH of flour. In Official methods of analysis of AOAC International (16th ed.). Arlington: AOAC International

2. ATHMASELVI, K.A.; KUMAR, C.; POOJITHA, P. 2017. Influence of temperature, voltage gradient and electrode on ascorbic acid degradation kinetics during ohmic heating of tropical fruit pulp. J. Food Meas. Charact. 11(1):144-155. https://doi.org/10.1007/s11694-016-9381-5

3. BENZIE, I.F.F.; STRAIN, J.J. 1996. The ferric reducing ability of plasma (FRAP) as a measure of "Antioxidant power": the FRAP assay. Anal. Biochem. 239(1):70-76. https://doi. org/10.1006/abio.1996.0292

4. BERNAL DE RAMÍREZ, I. 1998. Fruits and vegetables and their products. In: Food Analysis (3rd ed.). Bogotá: Academia Colombiana de Ciencias Exactas, Fisicas y Naturales. p.114-116.

5. BRAND-WILLIAMS, W.; CUVELIER, M.E.; BERSET, C. 1995. Use of a free radical method to evaluate antioxidant activity. LWT-Food Sci. Technol. 28(1):25-30. https://doi. org/10.1016/S0023-6438(95)80008-5

6. CAPOCASA, F.; SCALZO, J.; MEZZET'TI, B.; BATTINO, M. 2008. Combining quality and antioxidant attributes in the strawberry: The role of genotype. Food Chem. 111(4):872878. https://doi.org/10.1016/j.foodchem.2008.04.068

7. CASSIDY, A.; BERTOIA, M.; CHIUVE, S.; FLINT, A.; FORMAN, J.; RIMM, E.B. 2016. Habitual intake of anthocyanins and flavanones and risk of cardiovascular disease in men. Am. J. Clin. Nutrit. 104(3):587-594. https://doi. org/10.3945/ajcn.116.133132

8. CHANG, C.L.; CHEN, H.S.; SHEN, Y.C.; LAI, G.H.; LIN, P.K.; WANG, C.M. 2013. Phytochemical composition, antioxidant activity and neuroprotective effect of Crataegus pinnatifida fruit. South Afr. J. Bot. 88:432-437. https://doi. org/10.1016/j.sajb.2013.08.017

9. DA SILVA, L.M.R.; DE FIGUEIREDO, E.A.T.; RICARDO, N.M.P.S.; VIEIRA, I.G.P.; DE FIGUEIREDO, R.W.; BRASIL, I.M.; GOMES, C.L. 2014. Quantification of bioactive compounds in pulps and by-products of tropical fruits from Brazil. Food Chem. 143:398-404. https://doi. org/10.1016/j.foodchem.2013.08.001
10. DE SOUZA, V.R.; PEREIRA, P.A.P.; DA SILVA, T.L.T.; DE OLIVEIRA LIMA, L.C.; PIO, R.; QUEIROZ, F. 2014. Determination of the bioactive compounds, antioxidant activity and chemical composition of Brazilian blackberry, red raspberry, strawberry, blueberry and sweet cherry fruits. Food Chem. 156:362-368. https://doi.org/10.1016/j.foodchem.2014.01.125

11. ENVIRONMENTAL PROTECTION AGENCY-EPA. 2007. Method 3051A Microwave assisted acid digestion of sediments, sludges, soils, and oils. Washington: EPA.

12. FOOD AND AGRICULTURAL ORGANIZATION OF THE UNITED NATIONS-FAO. 2012. Alcance de las pérdidas y el desperdicio de alimentos. In: Pérdidas y desperdicio de alimentos en el mundo - Alcance, causas y prevención. Roma. p.4-10.

13. FIGUEIRA, T.R.; LOPES, A.C.S.; MODENA, C.M. 2016. Promoters and barriers to fruit and vegetable consumption among Health Academy Program's users. Rev. Nutr. 29(1):85-95. https://doi.org/10.1590/167898652016000100009

14. GASPARRINI, M.; FORBES-HERNANDEZ, T.Y.; GIAMPIERI, F.; AFRIN, S.; ALVAREZ-SUAREZ, J.M.; MAZZONI, L., MEZZETTTI, B.; QUILES, j.l.; BATTINO, M. 2017. Anti-inflammatory effect of strawberry extract against LPS-induced stress in RAW 264.7 macrophages. Food Chem. Toxicol. 102:1-10. https://doi.org/10.1016/ j.fct.2017.01.018

15. GRZELAK-BŁASZCZYK, K.; KARLIŃSKA, E.; GRZĘDA, K.; RÓJ, E.; KOŁODZIEJCZYK, K. 2017. Defatted strawberry seeds as a source of phenolics, dietary fiber and minerals. LWT-Food Sci. Technol. 84:18-22. https:// doi.org/10.1016/j.lwt.2017.05.014

16. HOLZWARTH, M.; KORHUMMEL, S.; CARLE, R.; KAMMERER, D.R. 2012. Evaluation of the effects of different freezing and thawing methods on color, polyphenol and ascorbic acid retention in strawberries (Fragaria $\times$ ananassa Duch.). Food Res. Int. 48(1):241-248. https://doi. org/10.1016/j.foodres.2012.04.004

17. HORVITZ, S.; CHANAGUANO, D.; AROZARENA, I. 2017. Andean blackberries (Rubus glaucus Benth) quality as affected by harvest maturity and storage conditions. Sci. Hort. 226:293-301. https://doi.org/10.1016/j.scienta.2017.09.002

18. INSTITUTO COLOMBIANO DE NORMAS TÉCNICAS Y CERTIFICACIÓN -ICONTEC. 1997. NTC 4106 Frutas frescas. Mora de castilla. Especificaciones. Bogotá, D.C. 
19. JOHNSON, M.H.; GONZALEZ DE MEJIA, E. 2016. Phenolic compounds from fermented berry beverages modulated gene and protein expression to increase insulin secretion from pancreatic $\beta$-cells in vitro. J. Agric. Food Chem. 64(12):2569-2581. https://doi.org/10.1021/acs. jafc.6b00239

20. LIU, Y.; LIU, Y.; TAO, C.; LIU, M.; PAN, Y.; LV, Z. 2018. Effect of temperature and $\mathrm{pH}$ on stability of anthocyanin obtained from blueberry. J. Food Measurement Characterization. 12(3):1744-1753. https://doi.org/10.1007/s11694-018$9789-1$

21. MINISTERIO DE AGRICULTURA Y DESARROLLO RURAL-MADR. 2018. Agronet Red de Información y Comunicación del Sector Agropecuario Colombiano. Disponible desde Internet en: http://www.agronet.gov. co/ (con acceso 29/05/2018).

22. OBÓN, J.M.; CASTELLAR, M.R.; ALACID, M.; FERNÁNDEZ-LÓPEZ, J.A. 2009. Production of a red-purple food colorant from Opuntia stricta fruits by spray drying and its application in food model systems. J. Food Eng. 90(4):471479. https://doi.org/10.1016/j.jfoodeng.2008.07.013

23. PILJAC-ŽEGARAC, J.; ŠAMEC, D. 2011. Antioxidant stability of small fruits in postharvest storage at room and refrigerator temperatures. Food Res. Int. 44(1):345-350. https:/ / doi.org/10.1016/j.foodres.2010.09.039

24. SAHIN, S.; SUMMU, S. 2006. Propiedades electromagnéticas. In: Propiedades físicas de los alimentos. Ankara. p.190-203.

25. TARAZONA-DÍAZ, M.P.; VIEGAS, J.; MOLDAO-MARTINS, M.; AGUAYO, E. 2010. Bioactive compounds from flesh and by-product of fresh-cut watermelon cultivars. J. Sci. Food Agric. 91(5):805-812. https://doi.org/10.1002/ jsfa. 4250
26. TARAZONA-DÍAZ, M.P.; AGUAYO, E. 2013. Assessment of by-products from fresh-cut products for reuse as bioactive compounds. Food Sci. Technol. Int. 19(5):439-446. https:/ / doi.org/10.1177/1082013212455346

27. U.S. DEPARTMENT OF AGRICULTURE-USDA. 2018. Food Composition Databases Show Foods List. Disponible desde Internet: https://ndb.nal.usda.gov/ndb/

28. VALENTE, A.; ALBUQUERQUE, T.G.; SANCHES-SILVA, A.; COSTA, H.S. 2011. Ascorbic acid content in exotic fruits: A contribution to produce quality data for food composition databases. Food Res. Int. 44(7):2237-2242. https://doi.org/10.1016/j.foodres.2011.02.012

29. VAN DE VELDE, F; GRACE, M.H.; ESPOSITO, D.; PIROVANI, M.É.; LILA, M.A. 2016. Quantitative comparison of phytochemical profile, antioxidant, and anti-inflammatory properties of blackberry fruits adapted to Argentina. J. Food Compost. Anal. 47:82-91. https://doi. org/10.1016/j.jfca.2016.01.008

30. VIGNONI, L.A.; CÉSARI, R.M.; FORTE, M.; MIRÁBILE, M.L. 2006. Determinación de índice de color en ajo picado. Inf. Tecnol. 17(6):63-67. https://doi.org/10.4067/S071807642006000600011

31. YANG, J.W.; CHOI, I.S. 2016. Comparison of the phenolic composition and antioxidant activity of korean black raspberry, Bokbunja, (Rubus coreanus Miquel) with those of six other berries. CyTA - J. Food. 15(1):110-117. https://doi. org/10.1080/19476337.2016.1219390 